\title{
A Hybrid Mean Value Involving the Two-Term Exponential Sums and Two-Term Character Sums
}

\author{
Liu Miaohua ${ }^{1}$ and Li Xiaoxue ${ }^{2}$ \\ ${ }^{1}$ Institute of Science, Air Force Engineering University, Xian, Shaanxi 710051, China \\ ${ }^{2}$ Department of Mathematics, Northwest University, Xian, Shaanxi 710127, China
}

Correspondence should be addressed to Li Xiaoxue; lxx20072012@163.com

Received 16 October 2013; Accepted 20 January 2014; Published 27 February 2014

Academic Editor: Olivier Bahn

Copyright ( 2014 L. Miaohua and L. Xiaoxue. This is an open access article distributed under the Creative Commons Attribution License, which permits unrestricted use, distribution, and reproduction in any medium, provided the original work is properly cited.

The main purpose of this paper is using the properties of Gauss sums and the estimate for character sums to study the hybrid mean value problem involving the two-term exponential sums and two-term character sums and give an interesting asymptotic formula for it.

\section{Introduction}

Let $q \geq 3$ be an integer and $\chi$ denotes a Dirichlet character mod $q$. For any integers $m$ and $n$ with $(m n, q)=1$, we define the two-term exponential sum $C(m, n, k ; q)$ and two-term character sum $N(m, n, \chi ; q)$ as follows:

$$
\begin{gathered}
C(m, n, k ; q)=\sum_{a=1}^{q} e\left(\frac{m a^{k}+n a}{q}\right), \\
N(m, n, k, \chi ; q)=\sum_{a=1}^{q} \chi\left(m a^{k}+n a\right),
\end{gathered}
$$

where $e(x)=e^{2 \pi i x}, \chi$ denotes a nonprincipal Dirichlet character $\bmod q$, and $k$ is a fixed positive integer.

These sums play a very important role in the study of analytic number theory, so they caused many number theorists' interest and favor. Some works related to $C(m, n, k ; q)$ can be found in [1-5]. For example, Cochrane and Zheng [1] show that

$$
|C(m, n, k ; q)| \leq k^{\omega(q)} q^{1 / 2}
$$

where $\omega(q)$ denotes the number of all distinct prime divisors of $q$.
On the other hand, the sums $N(m, n, k, \chi ; q)$ are a special case of the general character sums of the polynomials

$$
\sum_{a=N+1}^{N+M} \chi(f(a))
$$

where $M$ and $N$ are any positive integers and $f(x)$ is a polynomial. If $q=p$ is an odd prime, then Weil (see [6]) obtained the following important conclusion.

Let $\chi$ be a qth-order character $\bmod p$; if $f(x)$ is not a perfect $q$ th power $\bmod p$, then we have the estimate

$$
\sum_{x=N+1}^{N+M} \chi(f(x)) \ll p^{1 / 2} \ln p,
$$

where "«" constant depends only on the degree of $f(x)$. Some related results can also be found in [7-10].

Now we are concerned about whether there exists an asymptotic formula for the hybrid mean value

$$
\sum_{m=1}^{q-1}\left|\sum_{a=1}^{q-1} \chi\left(m a^{k}+a\right)\right|^{2} \cdot\left|\sum_{b=1}^{q-1} e\left(\frac{m b^{k}+b}{q}\right)\right|^{2} .
$$

In this paper, we will use the analytic method and the properties of character sums to study this problem and give a sharp asymptotic formula for (5) with $q=p$, an odd prime. That is, we will prove the following. 
Theorem 1. Let $p$ be an odd prime, let $\chi$ be any nonprincipal even character $\bmod p$, and let $\chi^{3} \neq \chi_{0}$ be the principal character $\bmod p$. Then we have the asymptotic formula

$$
\sum_{m=1}^{p-1}\left|\sum_{a=1}^{p-1} \chi\left(m a^{3}+a\right)\right|^{2} \cdot\left|\sum_{b=1}^{p-1} e\left(\frac{m b^{3}+b}{p}\right)\right|^{2}=2 p^{3}+E(p),
$$

where $E(p)$ satisfies the inequalities $-12 p^{2}-2 p \leq E(p) \leq$ $4 p^{2}-2 p$.

From this theorem we may immediately deduce the following.

Corollary 2. For any odd prime $p$ and any nonprincipal even character $\chi \bmod p$ with $\chi^{3} \neq \chi_{0}$, one has

$$
\sum_{m=1}^{p-1}\left|\sum_{a=1}^{p-1} \chi\left(m a^{3}+a\right)\right|^{2} \cdot\left|\sum_{b=1}^{p-1} e\left(\frac{m b^{3}+b}{p}\right)\right|^{2}=2 p^{3}+O\left(p^{2}\right) .
$$

In the theorem, we only consider the polynomial $f(x)=$ $m x^{3}+x$. For general polynomial $f(x)=m x^{k}+x^{h}$ with $k \geq 4$ and $1 \leq h<k$, whether there exists an asymptotic formula is complex problem for (5), it needs us to further study.

For general positive integer $q \geq 4$, whether there exists an asymptotic formula for (5) is also an interesting open problem.

\section{Several Lemmas}

To complete the proof of our theorem, we need the following several lemmas.

Lemma 1. Let $p$ be an odd prime and let $\chi$ be any nonprincipal even character $\bmod p$. Then for any integer $m$ with $(m, p)=1$, the identity

$$
\begin{aligned}
\sum_{a=1}^{p-1} \chi\left(m a^{3}+a\right)= & \frac{\tau\left(\chi_{1}\right) \tau\left(\bar{\chi}_{1}^{3}\right) \overline{\chi_{1}}(m)}{\tau(\bar{\chi})} \\
& \times\left(1+\left(\frac{m}{p}\right) \frac{\tau\left(\chi_{1} \chi_{2}\right) \tau\left(\bar{\chi}_{1}^{3} \chi_{2}\right)}{\tau\left(\chi_{1}\right) \tau\left(\bar{\chi}_{1}^{3}\right)}\right),
\end{aligned}
$$

where $(* / p)=\chi_{2}$ denotes the Legendre symbol and $\chi=\chi_{1}^{2}$.

Proof. Since $\chi(-1)=1$, there exists one and only one character $\chi_{1} \bmod p$ such that $\chi=\chi_{1}^{2}$. Thus, from the properties of Gauss sums we have

$$
\begin{aligned}
\sum_{a=1}^{p-1} \chi\left(m a^{3}+a\right) & =\frac{1}{\tau(\bar{\chi})} \sum_{a=1}^{p-1} \sum_{b=1}^{p-1} \bar{\chi}(b) e\left(\frac{b\left(m a^{3}+a\right)}{p}\right) \\
& =\frac{1}{\tau(\bar{\chi})} \sum_{a=1}^{p-1} \sum_{b=1}^{p-1} \bar{\chi}(b \bar{a}) e\left(\frac{b \bar{a}\left(m a^{3}+a\right)}{p}\right)
\end{aligned}
$$

$$
\begin{aligned}
& =\frac{1}{\tau(\bar{\chi})} \sum_{b=1}^{p-1} \bar{\chi}(b) e\left(\frac{b}{p}\right) \sum_{a=1}^{p-1} \chi(a) e\left(\frac{b m a^{2}}{p}\right) \\
& =\frac{1}{\tau(\bar{\chi})} \sum_{b=1}^{p-1} \bar{\chi}(b) e\left(\frac{b}{p}\right) \sum_{a=1}^{p-1} \chi_{1}^{2}(a) e\left(\frac{b m a^{2}}{p}\right) \\
& =\frac{1}{\tau(\bar{\chi})} \sum_{b=1}^{p-1} \bar{\chi}(b) e\left(\frac{b}{p}\right) \\
& \times \sum_{a=1}^{p-1} \chi_{1}(a)\left(1+\left(\frac{a}{p}\right)\right) e\left(\frac{b m a}{p}\right) \\
& =\frac{1}{\tau(\bar{\chi})} \sum_{b=1}^{p-1} \bar{\chi}(b) e\left(\frac{b}{p}\right) \\
& \times\left(\overline{\chi_{1}}(b m) \tau\left(\chi_{1}\right)\right. \\
& \left.+\overline{\chi_{1}}(b m) \chi_{2}(b m) \tau\left(\chi_{1} \chi_{2}\right)\right) \\
& =\frac{\overline{\chi_{1}}(m)}{\tau(\bar{\chi})}\left(\tau\left(\chi_{1}\right) \tau\left({\overline{\chi_{1}}}^{3}\right)\right. \\
& \left.+\left(\frac{m}{p}\right) \tau\left(\chi_{1} \chi_{2}\right) \tau\left({\overline{\chi_{1}}}^{3} \chi_{2}\right)\right) \\
& =\frac{\tau\left(\chi_{1}\right) \tau\left(\bar{\chi}_{1}^{3}\right) \overline{\chi_{1}}(m)}{\tau(\bar{\chi})} \\
& \times\left(1+\left(\frac{m}{p}\right) \frac{\tau\left(\chi_{1} \chi_{2}\right) \tau\left({\overline{\chi_{1}}}^{3} \chi_{2}\right)}{\tau\left(\chi_{1}\right) \tau\left({\overline{\chi_{1}}}^{3}\right)}\right) .
\end{aligned}
$$

This proves Lemma 1.

Lemma 2. Let $p$ be an odd prime, let $\chi$ be any nonprincipal even character mod $p, \chi=\chi_{1}^{2}$, and $\chi^{3} \neq \chi_{0}$, the principal character $\bmod p$. Then for any integer $m$ and any quadratic nonresidue $r \bmod p$ with $(m, p)=1$, we have the identity

$$
\begin{aligned}
\left|\sum_{a=1}^{p-1} \chi\left(m a^{3}+a\right)\right|^{2}= & 2 p+\left(\frac{m}{p}\right) \frac{\tau^{2}\left(\chi_{2}\right)}{2 p} \sum_{a=1}^{p-1}(\chi(a)+\bar{\chi}(a)) \\
& \times \sum_{b=1}^{p-1}\left(\frac{1-a^{2} b^{3}}{p}\right)\left(\frac{1-b}{p}\right) \\
& +\left(\frac{m}{p}\right) \frac{\tau^{2}\left(\chi_{2}\right)}{2 p} \\
& \times \sum_{a=1}^{p-1}\left(\chi_{1}(r) \chi(a)+\overline{\chi_{1}}(r) \bar{\chi}(a)\right) \\
& \times \sum_{b=1}^{p-1}\left(\frac{1-r a^{2} b^{3}}{p}\right)\left(\frac{1-b}{p}\right)
\end{aligned}
$$


Proof. From the properties of Gauss sums we have

$$
\begin{aligned}
\overline{\tau\left(\chi_{1}\right)} \tau\left(\chi_{1} \chi_{2}\right) & =\sum_{a=1}^{p-1} \bar{\chi}_{1}(a) \sum_{b=1}^{p-1} \chi_{1}(b) \chi_{2}(b) e\left(\frac{b-a}{p}\right) \\
& =\sum_{a=1}^{p-1} \bar{\chi}_{1}(a) \sum_{b=1}^{p-1} \chi_{2}(b) e\left(\frac{b(1-a)}{p}\right) \\
& =\tau\left(\chi_{2}\right) \sum_{a=1}^{p-1} \bar{\chi}_{1}(a)\left(\frac{1-a}{p}\right)
\end{aligned}
$$

So from (11) we have

$$
\begin{aligned}
& \frac{\tau\left(\chi_{1} \chi_{2}\right) \tau\left(\bar{\chi}_{1}^{3} \chi_{2}\right)}{\tau\left(\chi_{1}\right) \tau\left(\bar{\chi}_{1}^{3}\right)} \\
&=\frac{1 \overline{p^{2}} \overline{\tau\left(\chi_{1}\right) \tau\left(\bar{\chi}_{1}^{3}\right)} \tau\left(\chi_{1} \chi_{2}\right) \tau\left(\bar{\chi}_{1}^{3} \chi_{2}\right)}{=} \frac{\tau^{2}\left(\chi_{2}\right)}{p^{2}} \sum_{a=1}^{p-1} \bar{\chi}_{1}(a)\left(\frac{1-a}{p}\right) \sum_{b=1}^{p-1} \chi_{1}^{3}(b)\left(\frac{1-b}{p}\right) \\
&= \frac{\tau^{2}\left(\chi_{2}\right)}{p^{2}} \sum_{a=1}^{p-1} \bar{\chi}_{1}(a) \sum_{b=1}^{p-1}\left(\frac{1-a b^{3}}{p}\right)\left(\frac{1-b}{p}\right) \\
&= \frac{\tau^{2}\left(\chi_{2}\right)}{2 p^{2}} \sum_{a=1}^{p-1} \bar{\chi}(a) \sum_{b=1}^{p-1}\left(\frac{1-a^{2} b^{3}}{p}\right)\left(\frac{1-b}{p}\right) \\
&+\bar{\chi}_{1}(r) \frac{\tau^{2}\left(\chi_{2}\right)}{2 p^{2}} \sum_{a=1}^{p-1} \bar{\chi}(a) \sum_{b=1}^{p-1}\left(\frac{1-r a^{2} b^{3}}{p}\right)\left(\frac{1-b}{p}\right) .
\end{aligned}
$$

Note that $|\tau(\chi)|=\left|\tau\left(\chi_{1}\right)\right|=\left|\tau\left(\chi_{1}^{3}\right)\right|=\sqrt{p}$ and $\tau^{2}\left(\chi_{2}\right)=$ $\pm p$; from (12) and Lemma 1 we may immediately deduce the identity

$$
\begin{aligned}
\mid \sum_{a=1}^{p-1} \chi & \left.\left(m a^{3}+a\right)\right|^{2} \\
= & p \cdot\left|1+\left(\frac{m}{p}\right) \frac{\tau\left(\chi_{1} \chi_{2}\right) \tau\left(\bar{\chi}_{1}^{3} \chi_{2}\right)}{\tau\left(\chi_{1}\right) \tau\left(\bar{\chi}_{1}^{3}\right)}\right|^{2} \\
= & 2 p+\left(\frac{m}{p}\right) \frac{\tau^{2}\left(\chi_{2}\right)}{2 p-1} \sum_{a=1}^{p-1}(\chi(a)+\bar{\chi}(a)) \\
& \times \sum_{b=1}^{p-1}\left(\frac{1-a^{2} b^{3}}{p}\right)\left(\frac{1-b}{p}\right)+\left(\frac{m}{p}\right) \frac{\tau^{2}\left(\chi_{2}\right)}{2 p} \\
& \times \sum_{a=1}^{p-1}\left(\chi_{1}(r) \chi(a)+\overline{\chi_{1}}(r) \bar{\chi}(a)\right) \\
& \times \sum_{b=1}^{p-1}\left(\frac{1-r a^{2} b^{3}}{p}\right)\left(\frac{1-b}{p}\right) .
\end{aligned}
$$

This proves Lemma 2.
Lemma 3. Let $p$ be an odd prime, let $\chi$ be any nonprincipal even character $\bmod p, \chi=\chi_{1}^{2}$, and $\chi^{3} \neq \chi_{0}$, the principal character $\bmod p$. Then for any integer $m$ and any quadratic nonresidue $r \bmod p$ with $(m, p)=1$, one has the estimate

$$
\begin{aligned}
\mid \sum_{a=1}^{p-1}\left(\chi_{1}(r) \chi(a)+\overline{\chi_{1}}(r) \bar{\chi}(a)\right) \sum_{b=1}^{p-1}\left(\frac{1-r a^{2} b^{3}}{p}\right)\left(\frac{1-b}{p}\right) \\
+\sum_{a=1}^{p-1}(\chi(a)+\bar{\chi}(a)) \sum_{b=1}^{p-1}\left(\frac{1-a^{2} b^{3}}{p}\right)\left(\frac{1-b}{p}\right) \mid \leq 4 p .
\end{aligned}
$$

Proof. Let $n$ be any integer such that $(m n / p)=-1$ or $(m / p)+$ $(n / p)=0$. Then from Lemma 2 we have

$$
\left|\sum_{a=1}^{p-1} \chi\left(m a^{3}+a\right)\right|^{2}+\left|\sum_{a=1}^{p-1} \chi\left(n a^{3}+a\right)\right|^{2}=4 p .
$$

Note that $\left|(m / p)\left(\tau^{2}\left(\chi_{2}\right) / p\right)\right|=1$; applying (15) and Lemma 2 we have the estimate

$$
\begin{aligned}
& \mid \sum_{a=1}^{p-1}\left(\chi_{1}(r) \chi(a)+\bar{\chi}(r) \bar{\chi}(a)\right) \sum_{b=1}^{p-1}\left(\frac{1-r a^{2} b^{3}}{p}\right)\left(\frac{1-b}{p}\right) \\
& \quad+\sum_{a=1}^{p-1}(\chi(a)+\bar{\chi}(a)) \sum_{b=1}^{p-1}\left(\frac{1-a^{2} b^{3}}{p}\right)\left(\frac{1-b}{p}\right) \mid \\
& =\left.|| \sum_{a=1}^{p-1} \chi\left(m a^{3}+a\right)\right|^{2}-\left|\sum_{a=1}^{p-1} \chi\left(n a^{3}+a\right)\right|^{2} \mid \\
& \leq\left|\sum_{a=1}^{p-1} \chi\left(m a^{3}+a\right)\right|^{2}+\left|\sum_{a=1}^{p-1} \chi\left(n a^{3}+a\right)\right|^{2} \leq 4 p .
\end{aligned}
$$

This proves Lemma 3.

Lemma 4. Let $p>3$ be a prime. Then we have the identity

$$
\sum_{m=1}^{p-1}\left(\frac{m}{p}\right)\left|\sum_{a=1}^{p-1} e\left(\frac{m a^{3}+a}{p}\right)\right|^{2}=-\tau^{2}\left(\chi_{2}\right)\left(2+\left(\frac{3}{p}\right)\right)
$$

where $(* / p)=\chi_{2}$ denotes the Legendre symbol.

Proof. For any odd prime $p$ and integer $n$ with $(n, p)=1$, from Hua's book [11] (Section 7.8, Theorem 8.2) we know that

$$
\sum_{a=1}^{p}\left(\frac{a^{2}+n}{p}\right)=-1
$$


From this identity and the definition and properties of Gauss sums we have

$$
\begin{aligned}
\sum_{m=1}^{p-1}\left(\frac{m}{p}\right)\left|\sum_{a=1}^{p-1} e\left(\frac{m a^{3}+a}{p}\right)\right|^{2} \\
=\sum_{a=1}^{p-1} \sum_{b=1}^{p-1} \sum_{m=1}^{p-1}\left(\frac{m}{p}\right) e\left(\frac{m\left(a^{3}-b^{3}\right)+a-b}{p}\right) \\
=\sum_{a=1}^{p-1} \sum_{b=1}^{p-1} \sum_{m=1}^{p-1}\left(\frac{m}{p}\right) e\left(\frac{m b^{3}\left(a^{3}-1\right)+b(a-1)}{p}\right) \\
=\tau\left(\chi_{2}\right) \sum_{a=1}^{p-1} \sum_{b=1}^{p-1}\left(\frac{b^{3}\left(a^{3}-1\right)}{p}\right) e\left(\frac{b(a-1)}{p}\right) \\
=\tau\left(\chi_{2}\right) \sum_{a=1}^{p-1}\left(\frac{a^{3}-1}{p}\right) \sum_{b=1}^{p-1}\left(\frac{b}{p}\right) e\left(\frac{b(a-1)}{p}\right) \\
=\tau^{2}\left(\chi_{2}\right)\left(\sum_{a=1}^{p}\left(\frac{a^{2}+3}{p}\right)-1-\left(\frac{3}{p}\right)\right) \\
=\sum_{a=1}^{p-1}\left(\frac{\left(a^{3}-1\right)(a-1)}{p}\right) \\
=\tau^{2}\left(\chi_{2}\right)\left(\sum_{a=1}^{p}\left(\frac{4 a^{2}+4 a+4}{p}\right)-1-\left(\frac{3}{p}\right)\right)
\end{aligned}
$$

This proves Lemma 4.

\section{Proof of the Theorem}

In this section, we will complete the proof of our theorem. Note that the identities $\left|\tau\left(\chi_{2}\right)\right|^{2}=p$ and

$$
\begin{aligned}
\sum_{m=1}^{p-1}\left|\sum_{a=1}^{p-1} e\left(\frac{m a^{3}+a}{p}\right)\right|^{2} & \\
& =\sum_{m=1}^{p}\left|\sum_{a=1}^{p-1} e\left(\frac{m a^{3}+a}{p}\right)\right|^{2}-1 \\
& =\sum_{a=1}^{p-1} \sum_{b=1}^{p-1} \sum_{m=1}^{p} e\left(\frac{m\left(a^{3}-b^{3}\right)+a-b}{p}\right)-1 \\
& =\sum_{a=1}^{p-1} \sum_{b=1}^{p-1} \sum_{m=1}^{p} e\left(\frac{m\left(a^{3}-1\right)+b(a-1)}{p}\right)-1 \\
& = \begin{cases}p^{2}-p-1, & \text { if } 3 \dagger p-1, \\
p^{2}-3 p-1, & \text { if } 3 \mid p-1 .\end{cases}
\end{aligned}
$$

So from (20), Lemmas 2, 3, and 4, and noting that $\left|\tau\left(\chi_{2}\right)\right|^{2}=p$ we have

$$
\begin{aligned}
& \sum_{m=1}^{p-1}\left|\sum_{a=1}^{p-1} \chi\left(m a^{3}+a\right)\right|^{2} \cdot\left|\sum_{b=1}^{p-1} e\left(\frac{m b^{3}+b}{p}\right)\right|^{2} \\
& =2 p \cdot \sum_{m=1}^{p-1}\left|\sum_{c=1}^{p-1} e\left(\frac{m c^{3}+c}{p}\right)\right|^{2} \\
& +\frac{\tau^{2}\left(\chi_{2}\right)}{2 p} \sum_{a=1}^{p-1}(\chi(a)+\bar{\chi}(a)) \\
& \times \sum_{b=1}^{p-1}\left(\frac{1-a^{2} b^{3}}{p}\right)\left(\frac{1-b}{p}\right) \\
& \times \sum_{m=1}^{p-1}\left(\frac{m}{p}\right)\left|\sum_{c=1}^{p-1} e\left(\frac{m c^{3}+c}{p}\right)\right|^{2}+\frac{\tau^{2}\left(\chi_{2}\right)}{2 p} \\
& \times \sum_{a=1}^{p-1}\left(\chi_{1}(r) \chi(a)+\bar{\chi}_{1}(r) \bar{\chi}(a)\right) \\
& \times \sum_{b=1}^{p-1}\left(\frac{1-a^{2} b^{3}}{p}\right)\left(\frac{1-b}{p}\right) \\
& \times \sum_{m=1}^{p-1}\left(\frac{m}{p}\right)\left|\sum_{c=1}^{p-1} e\left(\frac{m c^{3}+c}{p}\right)\right|^{2} \\
& =2 p^{3}+E(p) \text {, }
\end{aligned}
$$

where $E(p)$ satisfies the inequalities $-12 p^{2}-2 p \leq E(p) \leq$ $4 p^{2}-2 p$.

This completes the proof of our theorem.

\section{Conflict of Interests}

The authors declare that there is no conflict of interests regarding the publication of this paper.

\section{Acknowledgments}

The authors would like to thank the referee for their very helpful and detailed comments, which have significantly improved the presentation of this paper. This work is supported by the NSF (11371291) of China.

\section{References}

[1] T. Cochrane and Z. Zheng, "Bounds for certain exponential sums," The Asian Journal of Mathematics, vol. 4, no. 4, pp. 757-773, 2000, Loo-Keng Hua: a great mathematician of the twentieth century.

[2] T. Cochrane and C. Pinner, "Using Stepanov's method for exponential sums involving rational functions," Journal of Number Theory, vol. 116, no. 2, pp. 270-292, 2006. 
[3] T. Cochrane and Z. Zheng, "Upper bounds on a two-term exponential sum," Science in China, vol. 44, no. 8, pp. 1003-1015, 2001.

[4] T. Cochrane and Z. Zheng, "Pure and mixed exponential sums," Acta Arithmetica, vol. 91, no. 3, pp. 249-278, 1999.

[5] T. Cochrane, J. Coffelt, and C. Pinner, "A further refinement of Mordell's bound on exponential sums," Acta Arithmetica, vol. 116, no. 1, pp. 35-41, 2005.

[6] D. A. Burgess, "On Dirichlet characters of polynomials," Proceedings of the London Mathematical Society, vol. 13, pp. 537$548,1963$.

[7] T. M. Apostol, Introduction to Analytic Number Theory, Springer, New York, NY, USA, 1976.

[8] A. Granville and K. Soundararajan, "Large character sums: pretentious characters and the Pólya-Vinogradov theorem," Journal of the American Mathematical Society, vol. 20, no. 2, pp. 357-384, 2007.

[9] W. Zhang and Y. Yi, "On Dirichlet characters of polynomials," The Bulletin of the London Mathematical Society, vol. 34, no. 4, pp. 469-473, 2002.

[10] W. Zhang and W. Yao, "A note on the Dirichlet characters of polynomials," Acta Arithmetica, vol. 115, no. 3, pp. 225-229, 2004.

[11] L. K. Hua, Introducetion to Number Theory, Science Press, Beijing, China, 1957. 


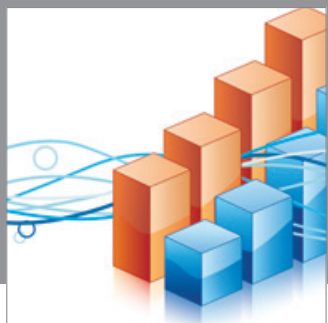

Advances in

Operations Research

mansans

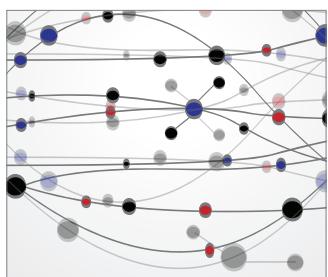

The Scientific World Journal
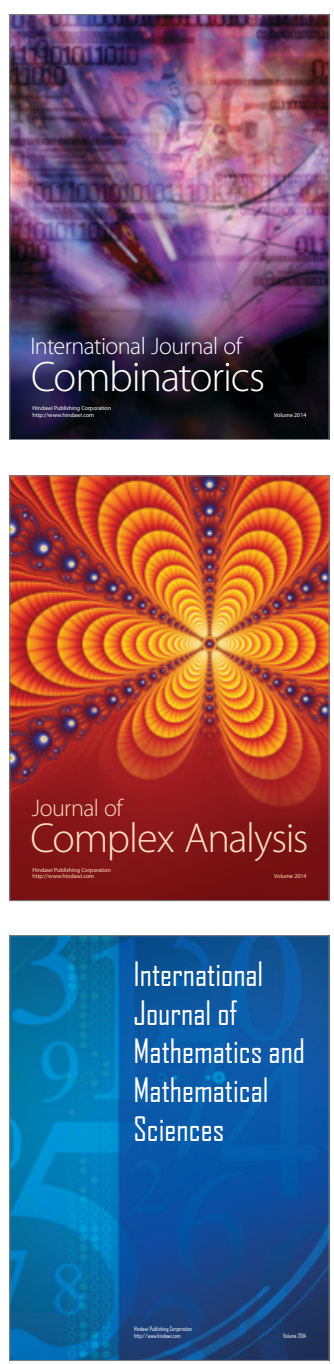
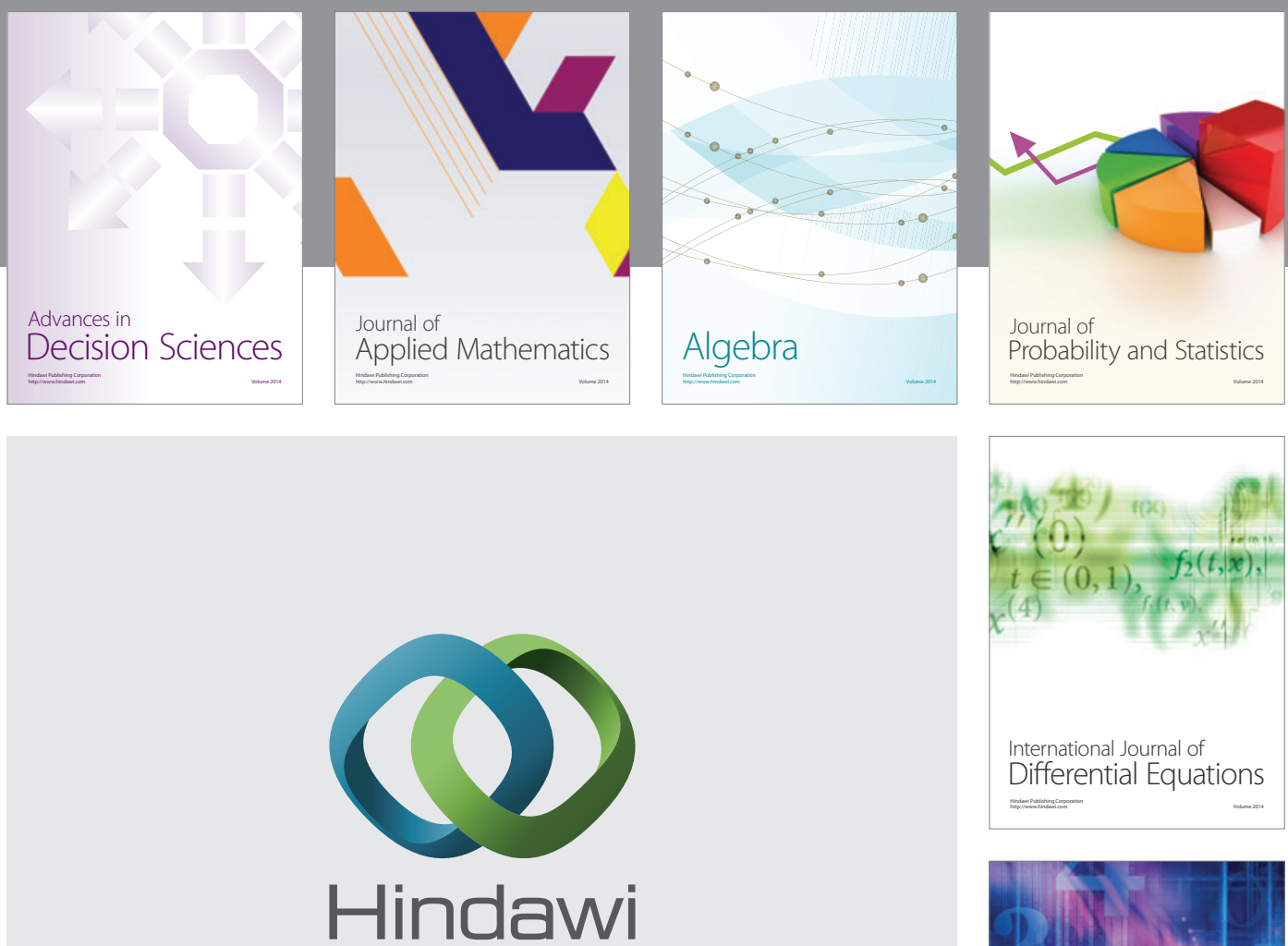

Submit your manuscripts at http://www.hindawi.com
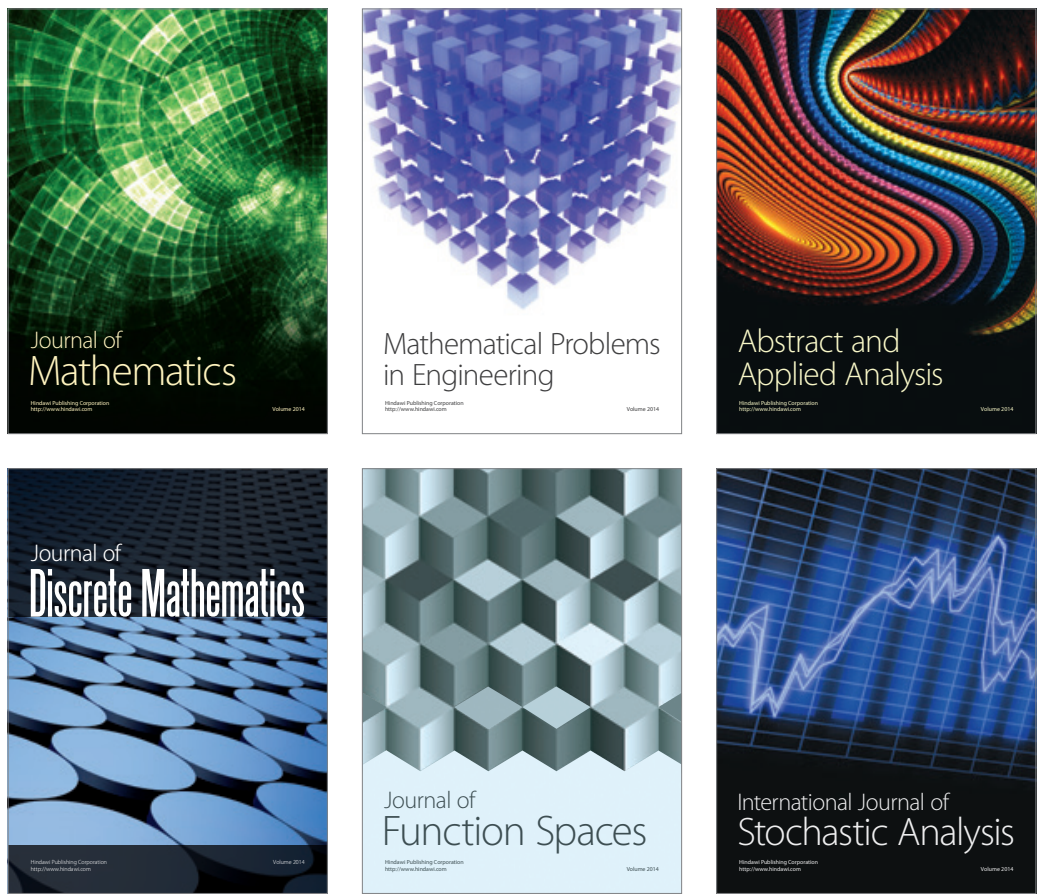

Journal of

Function Spaces

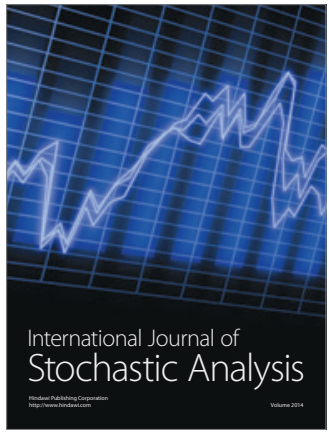

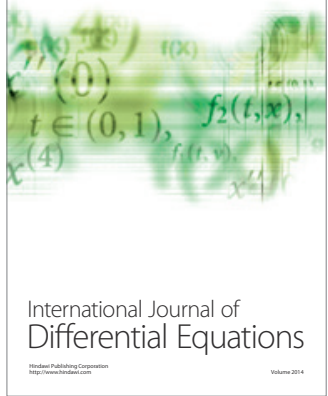
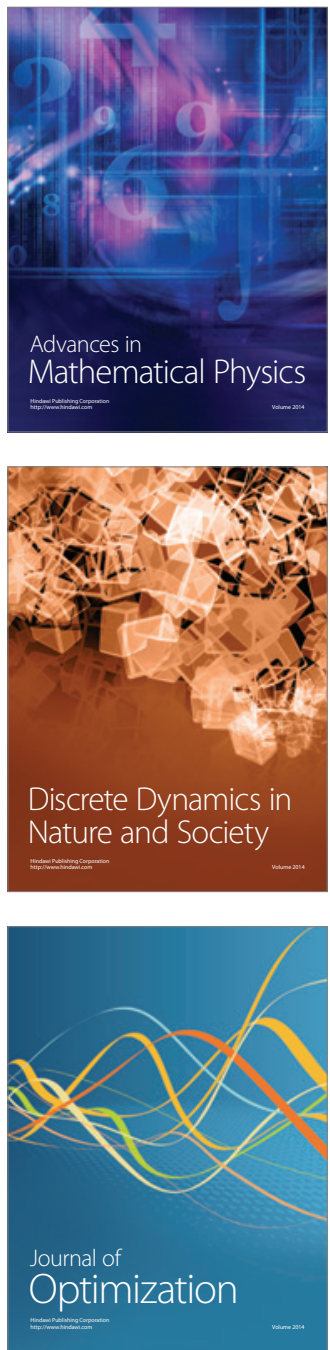